\title{
A spring network simulation in three dimensions for designing optimal crack pattern template to fabricate transparent conducting electrodes
}

\author{
SUPTI SADHUKHAN ${ }^{1}$, ANKUSH KUMAR ${ }^{2,3}$, GIRIDHAR U KULKARNI ${ }^{3}$, SUJATA TARAFDAR ${ }^{4}$ \\ and TAPATI DUTTA ${ }^{4,5, * \mathbb{D}}$ \\ ${ }^{1}$ Physics Department, Jogesh Chandra Chaudhuri College, Kolkata 700 033, India \\ ${ }^{2}$ Chemistry and Physics of Materials Unit, Jawaharlal Nehru Centre for Advanced Scientific Research, Bangalore 560 064, \\ India \\ ${ }^{3}$ Centre for Nano and Soft Matter Sciences, Jalahalli, Bangalore 560 013, India \\ ${ }^{4}$ Condensed Matter Physics Research Centre, Physics Department, Jadavpur University, Kolkata 700 032, India \\ ${ }^{5}$ Physics Department, St. Xavier's College, Kolkata 700 016, India \\ *Author for correspondence (tapati_dutta@sxccal.edu; tapati_mithu@yahoo.com)
}

MS received 24 October 2018; accepted 11 December 2018

\begin{abstract}
Desiccation cracks in colloids are of the order of $\mu \mathrm{m}$ width or less. Therefore, such connected crack networks in a desiccating colloidal film can provide a very fine template for the fabrication of transparent conducting surfaces by vapour deposition of a metal onto the crack network (Rao et al 2014 Adv. Mater. Interfaces 1 140009). The colloidal layer is removed leaving a connected metallic network invisible to the eye. So the surface becomes conducting, while retaining its transparency. The challenge lies in maximizing electrical conductivity while retaining the transparency as far as possible. An optimal combination of the system parameters, which affect the morphology of the crack network is necessary to meet this challenge. In this work, we simulate crack pattern in desiccating colloidal films in three dimensions using a spring network model. We look for the optimal combination of system parameters, such as film thickness, material stiffness and polydispersity, which can produce the best template for producing a conducting network on transparent surfaces.
\end{abstract}

Keywords. Crack pattern; desiccation; transparent electrode; spring network; simulation.

\section{Introduction}

Crack formation in various materials is a familiar sight and finds an important place in physics and engineering research. In recent times, the study of desiccation cracks [1] has become specially important as technical applications of intentionally designed crack patterns are coming up [2]. A cleverly generated crack network can be used as an important lithography template to fabricate a metal wire network on a transparent substrate for transparent conducting electrode (TCE) applications. What would be the most satisfactory approach towards choosing the right materials and conditions for the perfect crack template, suited for such a specific application? Ideally, a theoretical model incorporating the relevant material properties and ambient conditions which could predict the final crack pattern should give the answer to this problem. However, a full theoretical analysis of such a complex system is too much to be practicable. In this paper, we take a more feasible route and simulate the drying sample through a simple model consisting of a lattice of nodes connected by springs. The choice of an underlying lattice of regular geometry often results in crack patterns that reflect, to some degree, the geometry of the lattice. A cuboidal lattice of springs connecting nodes yields cracks that meet at right angles much like cracks do in many cases. Spring network models using a triangular lattice of springs have been earlier reported by ref. [3] to simulate fractal crack patterns where the cracks meet at $\sim 120^{\circ}$. Fracture anisotropy intrinsically exists in the regular lattice models employing only the nearest neighbours. Including the influence of more neighbours on a single node lessens the anisotropy but is computationally expensive. In the random network models, since each connecting spring (i.e., bond) has different properties, certain scaling technique is required to achieve elastic uniformity. Also, some other type of interaction, such as shear spring is introduced so as to remove the limitation on the effective Poisson's ratio. This artefact is almost absent if lattice free models are used for crack simulation [4,5]. Spring network models have been used by many groups to create realistic crack patterns generated under different conditions [6-11]. However, these works reported cracks formed in materials having a single solute-solvent composition.

There are some reports in the existing literature on efforts to model the crack physics using a spring network model 
addressing cracking of composite materials [12,13], the effect of adhesion, and competition between cracking and peeling in two-dimensional (2D) models [14,15]. Vogel et al [16] and Khatun et al [10] introduced topological concepts to analyse crack patterns. Recently, some authors have tried to relate the material properties of the medium with crack formation [17]. However, accurate prediction of crack morphology on the basis of physical and ambient conditions is yet to be achieved. By establishing a correspondence between parameters used in the theoretical model and physical properties of the cracking system, we expect to identify the system and conditions, which will produce the ideal crack pattern.

In the present paper, we study crack patterns formed by desiccation in a 3D model based on a spring network. The objective is to study crack network morphology with special relevance to optimizing electrical conductivity, while retaining the transparency of a transparent substrate. In the next section, the experimental method, which generates the TCE utilizing a crack network formed on a colloidal layer, is discussed in some details. In section 2.2, the simulation algorithm and its implementation are given, and in the following section 3 , simulation results, focussing on the role of different parameters and drying conditions, are discussed. Finally, we compare simulation results with experiments and discuss how the simulations can help in choosing materials and fixing appropriate conditions to produce a transparent conducting surface (TCS) with desired characteristics in section 4 .

\section{Methods}

\subsection{Experimental method for creating TCE}

The experimental results, which are compared with the present simulation, have been recorded for the following system. $\mathrm{TiO}_{2}$ nanoparticles mixed in appropriate proportions of methanol/ethanol form a suspension through rigorous ultrasonication. This suspension is then drop coated on various substrates. The suspension when allowed to desiccate in the air forms cracks. Au metal is then vapour deposited at this stage. The metal can deposit on the substrate only if it finds a straight vertical channel. $\mathrm{TiO}_{2}$ is washed in water with mild sonication leaving behind the metallic mesh on the substrate. This mesh serves as the template for TCE $[18,19]$. The metal network with a width of a few $\mu \mathrm{m}$ is invisible to the eye, but with good connectivity, it renders the surface conducting. A pattern with wider and denser cracks gives higher conductivity to the surface, but compromises on the transparency. Thus, there is a trade-off between conductance and transmittance, so identifying materials and techniques to optimize high conductivity and good transparency is essential. Crack networks with specific geometry are required for such applications [20,21]. The challenge is to identify appropriate materials and conditions to create the most efficient network. Techniques, such as variation of drying temperature, film thickness, repeated wetting-drying cycles [22], etc. have been explored for tuning the crack geometry.

\subsection{The simulation procedure}

2.2a Basic algorithm: Our system consists of a regular arrangement of nodes on a cuboidal system of size $L \times L \times H$, of side length $L$ and height $H$. Though the cuboidal lattice geometry makes the system quite restrictive, considering that this is a $3 \mathrm{D}$ composite system, we consider this as a good starting point. The cuboidal lattice mimics the thin film, the nodes in the lattice represent particles of the film, while the distance between the nodes represents the range of the force of interaction between the particles of the system. The spring properties represent the elastic interaction between a particle (node) and its neighbours. As we consider the system to be homogeneous and isotropic with arbitrary Poisson's ratio, we assume the spring constants and nodal spacings to be identical. For most part of the study, we have considered $L=50$ units for limitations on computational time. A few images are taken for $L=200$. $H$ has been varied from 1 to 12 units. We keep $L \gg H$ to replicate the experimental set up where the corresponding dimensions are $5 \times 5 \mathrm{~cm}^{2}$ in lateral size and $20 \mu \mathrm{m}$ in height. The edge boundaries of the system are free to move under the action of forces. The nearest neighbour nodes are connected by Hookean springs vertically and horizontally except for the lowest layer, which has only the vertical spring attachment (figure 1).

The lowest layer of nodes is distinct from all the other nodes as they represent the substrate on which the film is deposited. The vertical springs attached to the lowest layer of nodes represent adhesion of the material to the substrate. Other nodes are connected to their nearest neighbours by vertical and horizontal springs, which represent cohesion among particles of the desiccating material. Each spring has a natural length, which is the length under force-free condition (figure 2a). The desiccation of the material is realized through a reduction of the natural length of the springs. Reduced natural length implies that the springs are no longer in force-free condition, but are strained. Each spring is attributed with a stiffness $Y$ and a breaking threshold $S$. Both these parameters characterize the substance undergoing desiccation. $Y$ is related to elastic properties of the substance. The breaking threshold signifies the maximum permissible accumulated strain beyond which a stretched spring breaks. Initially, the springs have a unit natural length. In this model, we only allow the breaking of the springs representing cohesion in the material. The lowest layer of nodes may be permitted to slip until a less strained configuration is reached [23]. For the present study, we have allowed no slip. Desiccation is implemented by reduction of the natural length of the springs with time according to the rule

$$
d_{n+1}=d_{n}\left(1-b / r^{n}\right) .
$$




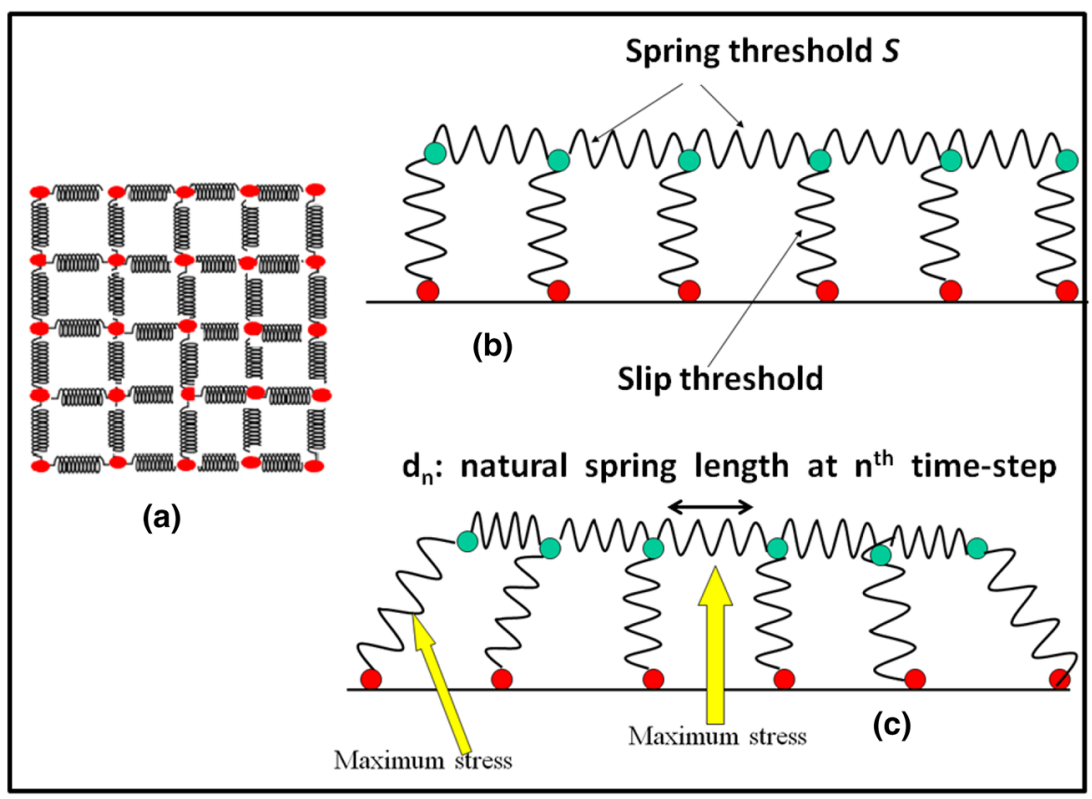

Figure 1. (a) Arrangement of nodes (red) and springs on any layer. (b and c) Schematic diagram of strain development in spring network. Nodes represent colloidal particles and spring represents interaction.

Here, $d_{n}$ and $d_{n+1}$ are the natural lengths of the springs at successive time steps $n$ and $n+1$, respectively, while $b$ and $r$ are constants. The form in equation (1) is integrated to the final form given in equation (2), which we use in the present paper

$$
d_{n}=\exp \left(b r^{-n} / \ln (r)\right) .
$$

We have normalized $d_{n}$ by $d_{0}$, the natural length at $n=0$. With increasing $n, d_{n}$ decreases. At a high $n$, the difference in natural lengths for two consecutive time steps, viz. $d_{n}$ and $d_{n+1}$, becomes negligible and the system is said to have saturated to $d_{\mathrm{min}}$. Experiments measuring the rate of the weight reduction of the drying colloid closely fit the power law in equation (2). The change in the weight almost saturates at $65-75 \%$ of the initial mass. Accordingly, we have chosen $b$ and $r$ such that $d_{\min }$ is $65-75 \%$ of the initial $d_{0}$ [24].

Each time when the desiccation rule is employed, stress builds up in the system. We have assumed that a node experiences force only due to its nearest neighbours. Each strained node is allowed to move under the action of the force, see figure $2 \mathrm{~b}$. The displacement between the $n$th and the $(n+1)$ th time-step is implemented using a simple form of Verlet's algorithm according to the relation $r_{n+1}=r_{n}+f \delta t^{2}$. Here $f$ is the force acting on the concerned node and $\delta t$ is the time during which the node moves. $\delta t$ is judiciously assigned a value of 0.005 unit. A very small $\delta t$ increases cumulative error for a finite time-step, while a large value is in conflict with the assumption that force acting in the system remains constant for the time span. After the system relaxes, the strains in all the springs except the layer attached to the substrate are determined. The spring with the largest strain is broken, if it exceeds the breaking threshold. The processes of (a) desiccation, (b) displacement of the nodes under the resultant force, followed by (c) breaking of the springs on satisfying the requisite condition is iterated, until the system saturates as mentioned above [14,15]. A flowchart of the algorithm followed in a single time step is shown in figure 3 .

The system is then checked for straight vertical crack connectivity spanning from the topmost layer to the lowest layer. This is done to mimic the experimental procedure in which metal vapour is deposited on the film system. Metal can only deposit on the substrate, if a vertical straight crack from the top to the bottom layer is present.

If one or more such vertical cracks are obtained then, the simulated sample film is checked for horizontal crack connectivity spanning across the substrate plane. This is to check that the metallic crack network on the substrate after the colloidal material is washed away, spans the system ensuring that the surface has acquired a non-zero conductivity. The properties of the crack network obtained on varying the simulation parameters are determined. The relevant properties are crack spacing (or crack density), crack width and connectivity as well as the fill factor (defined as the fraction of the total area covered by cracks). Together, they determine the conductivity and transparency. Conductivity and transparency have to be optimized so as to simultaneously maximize the both. Obviously, a higher conductivity, requiring more densely spaced wider cracks will tend to increase crack fill factor and hence, reduce transparency. 


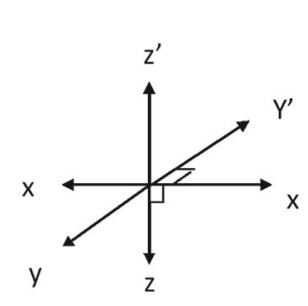

(a) Initial: Regular cube

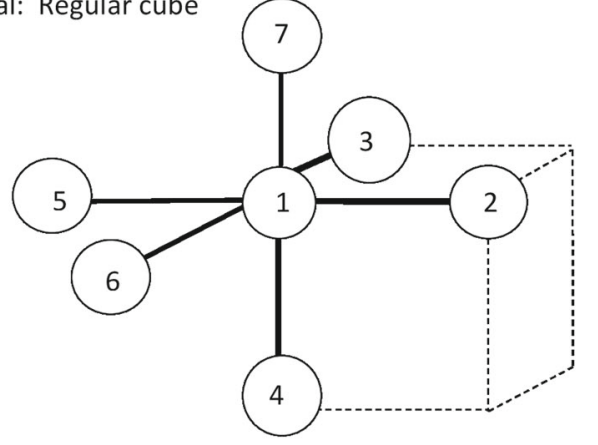

(b) After desiccation: distorted cube

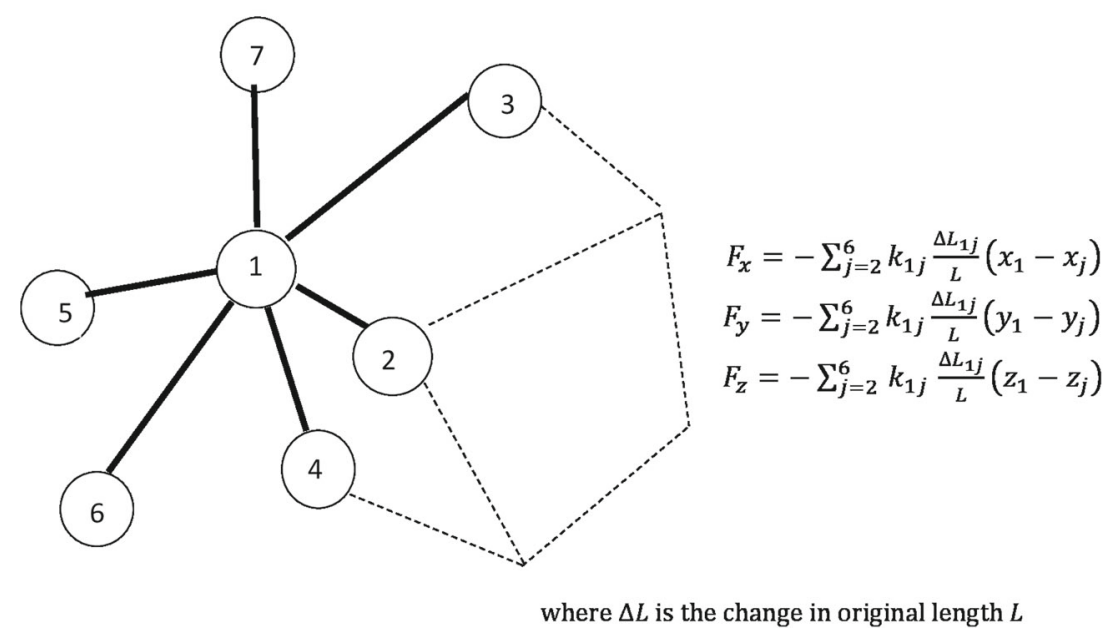

Figure 2. (a) Arrangement of reference axes. Schematic diagram of spring connection between a single node and its nearest neighbours. Nodes represent colloidal particles and spring represents interaction. (b) The deformation of springs after desiccation. The stress in each spring contributes to a force component in each direction.

2.2b Conductivity calculation: The conductivity is determined using Laplace's equation. To solve for conductivity, the crack network on the substrate is discretized by spreading on it a virtual mesh of square cells. The portion of the mesh covered by the cracks corresponds to the vacant cells, while the rest correspond to occupied cells. We consider each pair of adjacent vacant cells to be connected by a unit resistor and unit potential difference is applied to the sample under study. The potential distribution in the vacant cells is determined by solving Laplace's equation:

$$
\nabla^{2} V(x, y, z)=0 .
$$

Using the finite difference scheme, this reduces to

$$
\frac{V_{i-1, j}-2 V_{i, j}+V_{i+1, j}}{(\Delta x)^{2}}+\frac{V_{i, j-1}-2 V_{i, j}+V_{i, j+1}}{(\Delta y)^{2}}=0 .
$$

$\Delta x$ is the spatial discretization unit. This unit is equal to the side length of a grid cell. In our case, $\Delta x=\Delta y=1$. Rewriting the above equation:

$$
V_{i, j}^{n+1}=\frac{1}{p}\left[V_{i-1, j}^{n}+V_{i+1, j}^{n}+V_{i, j-1}^{n}+V_{i, j+1}^{n}\right] .
$$

Here, $p$ is the sum of the nearest neighbouring vacant cells. The Gauss-Seidel iterative method is employed to solve equation (5). At $n=0, V^{n}=0$ in all the cells. A steady state is attained when the potential distribution does not vary with time. The tolerance limit for achieving steady state is set to $10^{-7}$. At steady state, the sum of the potential differences of the vacant cells at the inlet or the outlet is numerically equal to the conductance as the potential difference across the sample is unity [25]. Since the size of the sample along the horizontal directions is fixed $(L \times L)$, the conductance and conductivity scale linearly by a fixed factor.

2.2c Estimation of transparency: The occupied peds, i.e., the regions of the colloidal layer, which are intact after crack 


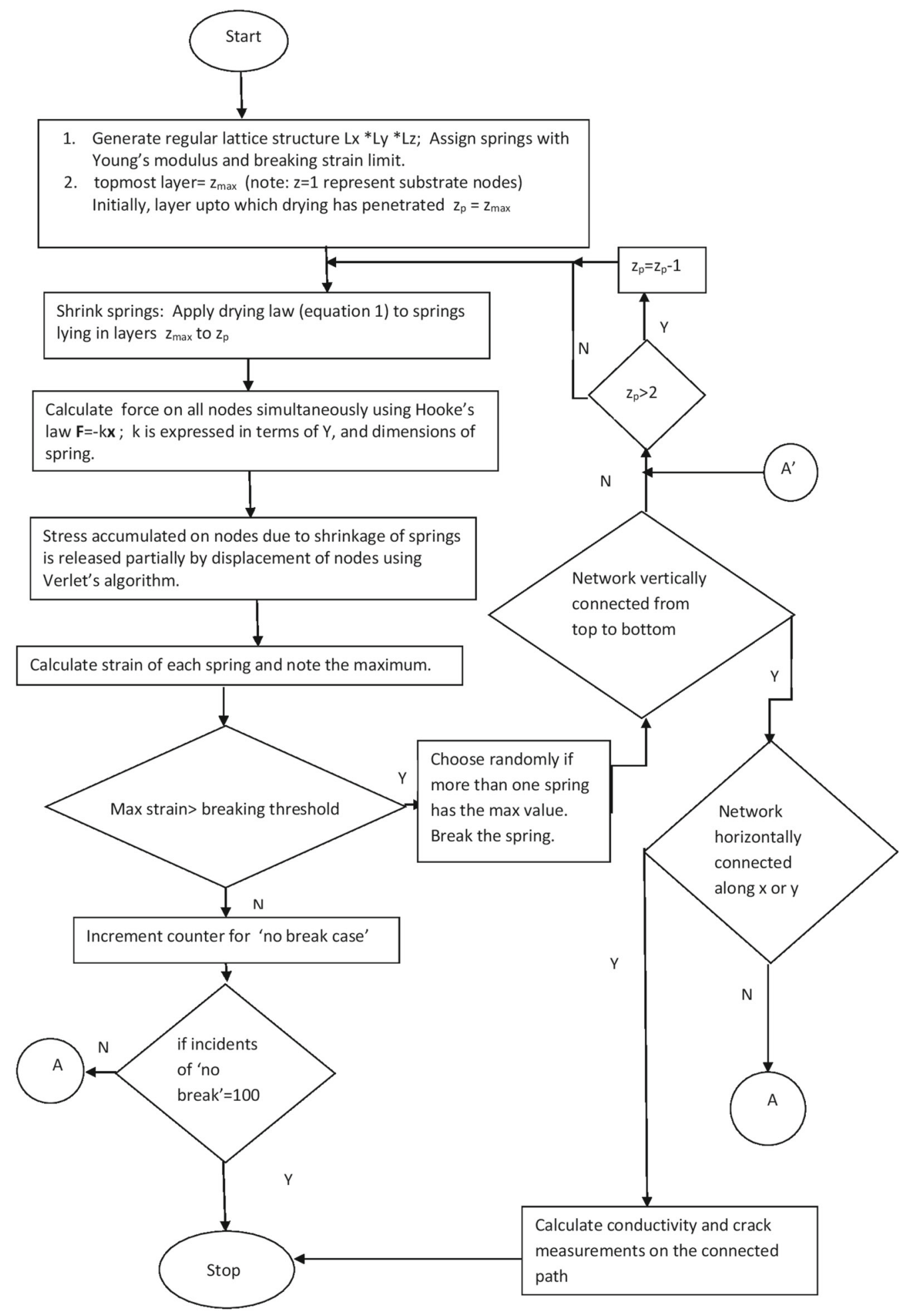

Figure 3. Flowchart of a single simulation time step. The point $\mathrm{A}^{\prime}$ is the sink to which the two $\mathrm{A}$ nodes re-join to begin the next cycle of iteration.

formation, will be transparent since the colloidal layer is washed away after vapour deposition. So, the greater the area fraction of these regions, the higher is the transparency of the TCS. In the figures of crack patterns (figure 4), these regions are shown in black. The fraction of the total area occupied by cracks is designated as the fill factor $\phi$ and used 
as a quantitative indicator of the transparency . In general, therefore, our goal is to maximize $\phi$, while simultaneously maximizing the conductivity $(\sigma)$. However, it must be remembered that very wide connected cracks may give high $\sigma$ as well as $\phi$, but still not suitable for TCE application, as too wide metal-deposit strips will become visible to the eye. So, the actual crack width is also important and must be $\sim \mathrm{nm}$ to $\mu \mathrm{m}$.

\subsection{Correspondence between model parameters and physical properties}

The parameters we have used in the model can be listed as follows: (i) spring network parameters: spring constant $Y$ and spring breaking threshold $S$; (ii) desiccation parameters: $b$ and $r$; and (iii) sample dimensions thickness $H$ and lateral size $L$. In this study, we have not allowed the slipping movement of the nodes, assuming the substrate has a rough texture. $L$ has been kept constant at 50 .

2.3a Spring network parameters: Let us first consider the system to be an elastic material. In this case, the spring constant or stiffness $Y$ physically represents the stress/strain ratio or Young's modulus of the material. The strain in our case arises due to a reduction in the natural length of a spring by drying. For normal elastic solids, Young's modulus can be measured by standard methods [26,27]. However, since the desiccating material is a visco-elastic colloidal suspension or gel, it is difficult to define or measure Young's modulus directly. Nano-indentation techniques may be applied, if the gel is somewhat stiff.

In the colloidal suspension, one may think of an attractive interaction between two particles (say spherical) through a liquid bridge formed by the solvent [1]. According to Goehring et al [1], the colloidal particles are bound by van der Waals interaction, whose strength depends on the dielectric properties of both particle and solvent. In this case, the Hamaker constant of the fluid-solid combination would be the measurable physical property associated with the spring parameter. The attraction or adhesion between the colloid and the substrate can be similarly represented, using van der Waals interaction between the substrate and the colloid.

The breaking threshold $S$ of the springs in the model determines when the spring actually breaks, on being stretched to a certain limit. In fracture literature, this is the fracture toughness related to the stress intensity factor [1,28,29]. It is also a measurable parameter for normal solids and may be accessible through indentation experiments in stiffer gels or pastes. In materials with plasticity or ductile materials, the stress at the tip of a crack is dissipated over a large region (may be of macroscopic size). Hence, the tendency to break or fail is much less. In a brittle material, however, the process zone with high stress intensity is microscopic causing breaking of molecular bonds. For example, in polymers, the plasticity is extremely high, whereas in a brittle clay suspension, it is low. The effect of adding increasing fractions of polymer to an aqueous Laponite ${ }^{\circledR}$ water gel was clearly demonstrated in experiments and simulation [13]. Cracks reduced drastically as a polymer with high breaking stress was added and for a 50-50 clay-polymer composite, no sample spanning crack formed at all.

2.3b Desiccation parameters: The empirical law, equation (1), is framed such that desiccation reduces with time and finally, the natural length of the spring saturates to $d_{\min }$ on complete drying. During desiccation, when a little solvent evaporates, the particles are drawn closer together, as we envisage in our model by shrinking of the springs. The parameters $b$ and $r$ control this process. Physically, the rate of shrinking depends on (i) the ambient conditions - relative humidity and temperature and (ii) the nature of the drying paste, e.g., the volatility of the solvent and surface tension of the suspension. The parameters $b$ and $r$ can be interpreted as representing these two distinct aspects of drying behaviour as follows.

Equation (1) can be reframed as

$$
\frac{d_{n+1}-d_{n}}{d_{n}}=-\frac{b}{r^{n}} .
$$

Here, the left-hand side is the fractional reduction in length at the $n$th time step. On the right-hand side, as $b$ increases desiccation by a constant amount per time step, independent of how long the drying has been continuing. The parameter $b$ represents the effect of the ambient conditions, assuming that the contribution from this effect remains the same for any material, under identical drying conditions. The denominator on the right-hand sid of equation (6) depends on how long the sample has been drying. In desiccation experiments, one sees that shrinking of the sample volume with time does not continue at the same rate, but decreases with time, ultimately reaching saturation as already mentioned. The denominator $r^{n}$ ensures that saturation is approached after a long enough time interval. How much the sample shrinks up to saturation depends on the material which is drying. So, $r$ represents a material characteristic related to drying. A smaller $r$ leads to more shrinking, but ultimate saturation of the sample volume at some large $n$.

The real system being extremely complex, there may be interdependence between the parameters, however, the above discussion can be taken as an approximate guideline.

\section{Results}

In this section, we present the results of the simulation, which bring out the role of the different input parameters and simulation model parameters controlling crack geometry, varying each individually. The effect of variation of real input parameters, such as film thickness $H$ can be compared directly with experiments, but as discussed earlier in section 2.3, 


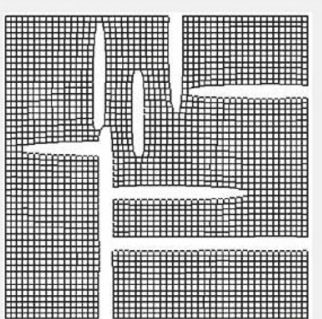

(a)

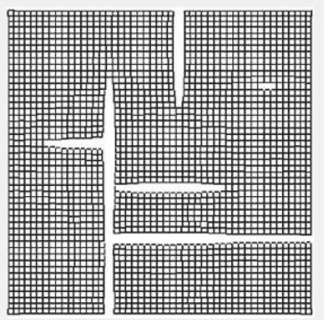

(d)

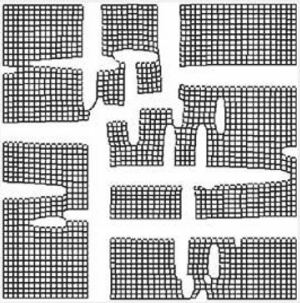

(b)

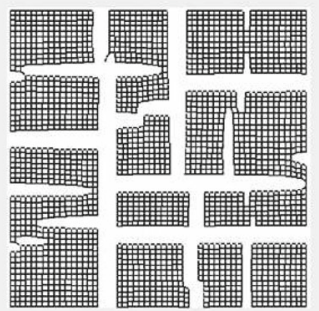

(e)

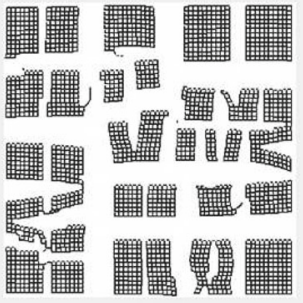

(c)

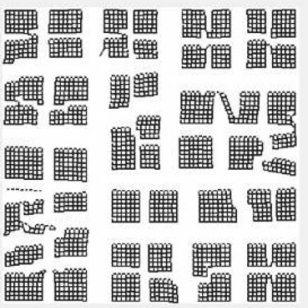

(f)

Figure 4. Crack development with time. The white regions represent the cracked area and the black represents the film. (a-c) Images of the topmost layer. (d-f) Images of the bottom layer.

other model parameters may depend on each other and assign one to one correspondence between simulation variables and measurable material properties could lead to an over simplification.

We specify a standard set of parameters given in table 1 . The limits of variation of each parameter are also tabulated here. When a particular parameter is varied, the remaining parameters are assigned constant values in the first row of table 1.

Figure 4 shows the time evolution of a typical configuration for a sample of size $50 \times 50 \times 4$ units. The formation of cracks on the topmost and bottom layers at certain intervals of time, and their final state when desiccation is complete are shown. Not all the cracks formed on the topmost layer are connected to the bottom layer at the final state. Figure 5 highlights the area on the base layer connected to the topmost layer. The simulated crack patterns also show hierarchical structures [30] and dangling cracks as typically seen in the real scenario.

\subsection{Effect of film thickness}

Figure $6 \mathrm{a}$ and $\mathrm{b}$ shows the experimental images and variation of conductivity $\sigma$ with thickness $H$ of the sample [19]. Figure $6 \mathrm{c}$ shows the top surface view of a typical simulated crack pattern on a sample with height $H$ varying from 4 to 12 units, after complete drying. The images are taken once the springs reach saturation, ensuring complete drying. These compare very well with the experimental images of actual samples in experiments (figure 6a). According to simulation

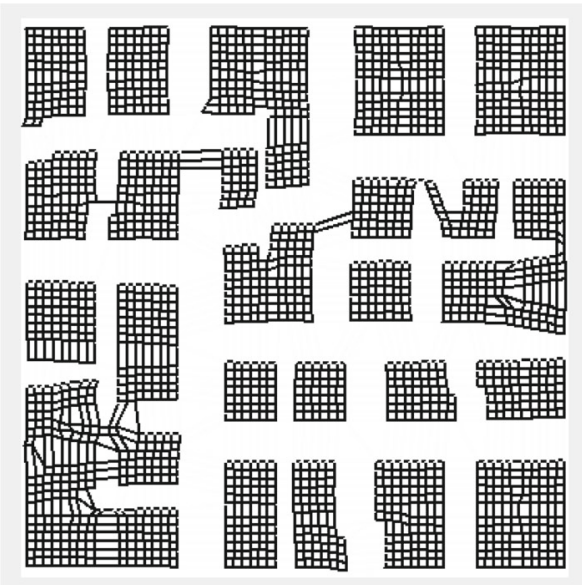

Figure 5. The conducting path (white) on the lowest layer.

results (figure 7), $\sigma$ is maximum for the minimum thickness in the range considered. In general, cracks appear at a certain minimum sample thickness known as the critical cracking thickness $H_{\mathrm{CCT}}[10,12,31]$. These may be threepronged star-like cracks, disjoint from each other. But with increasing thickness after a value $H_{0}>H_{\mathrm{CCT}}$, a connected network of cracks appears. Once this happens, crack spacing increases, being of the order of the thickness. This would lead to a decrease in $\sigma$ for $H \geq H_{0}$. So, the simulation result is realistic. 

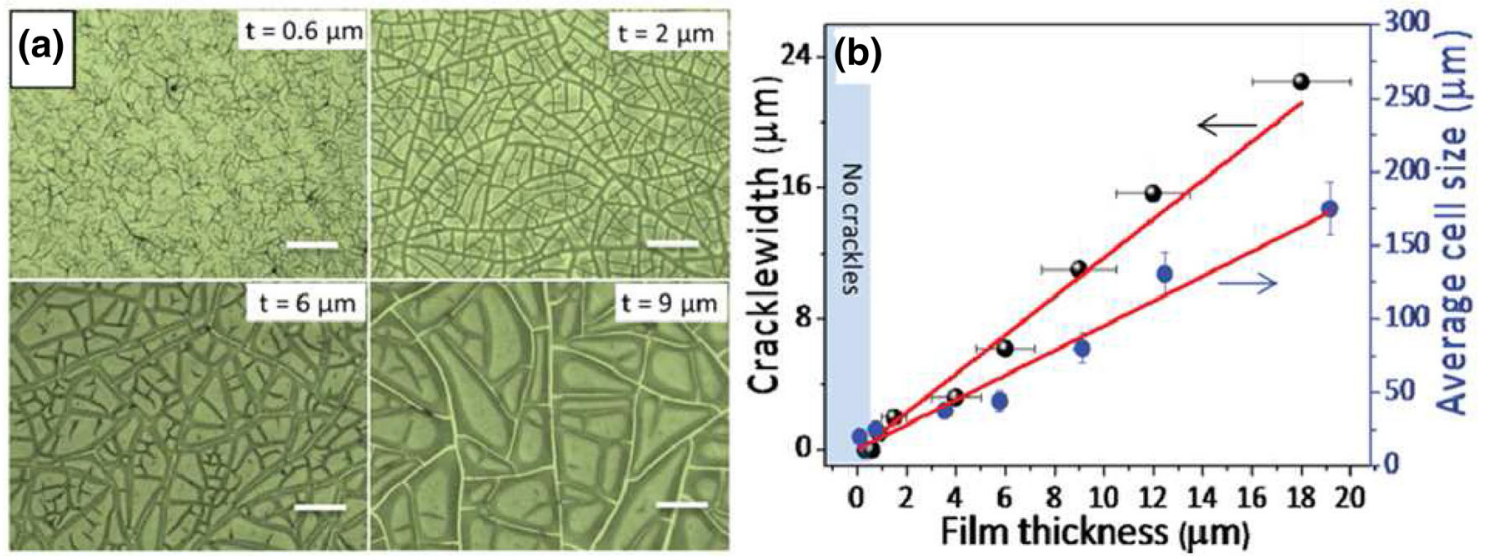

(c)

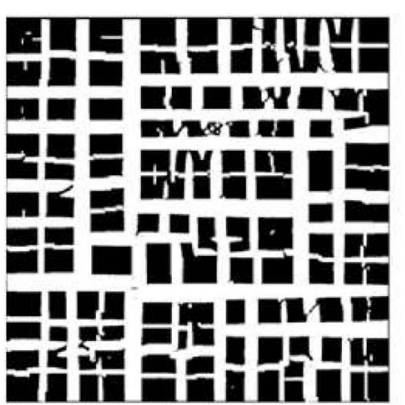

Thickness $=3$

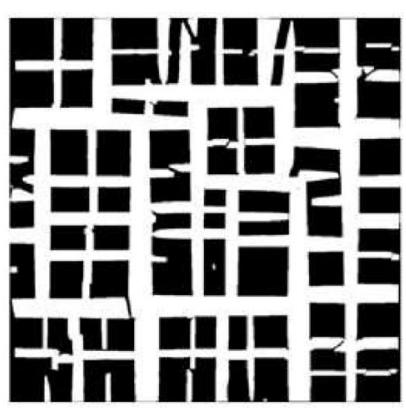

Thickness $=5$

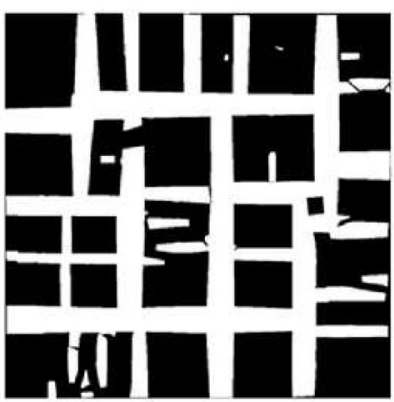

Thickness 10

Figure 6. (a) Experimental variation of crack pattern with film thickness, (b) variation in crack width and crack spacing with film thickness. Reproduced with permission from ref. [19], Copyright 2014 Wiley-VCH Verlag GmbH \& Co. (c) Simulated images for variation of film thickness for a typical sample.

\subsection{Effect of varying spring stiffness $Y$}

Figure 8 shows the view of the topmost layer of a set of simulations where $Y$ has been varied. In the simulation, this has been put into effect by varying the elastic modulus of the connecting springs of the network. Figure 9 shows that on the whole both conductivity and crack fill factor show a maximum at the lowest value $Y=1$ (arbitrary units), while the crack width and crack spacings increase with $Y$. The conductivity $\sigma$ vs. $Y$ graph, however, shows interesting behaviour. As $Y$ increases from 1 to 5 (arbitrary units), $\sigma$ initially decreases with increasing $Y$ and then increases to a distinct peak at $Y=3$ units before falling off again. At the peak in $\sigma$ at $Y=3$, the fill factor $\phi$ has dropped quite low. So, an ideal optimization of conductivity and transparency may be achieved for a material with $Y \sim 3$. This result also brings out a significant feature of network conductivity, namely, that the connectivity between strands of the network may override the effect of widths and lengths of individual strands. Figure 9 shows that in the region $Y \sim 3$ crack width is slightly higher, but crack spacing is considerably higher, accounting for the low fill factor. The good crack network connectivity in this region must be the deciding factor here, leading to high $\sigma$ with low $\phi$.

Careful scrutiny of figure 8 shows that for the lowest $Y$, though the network appears dense, many junctions have a break in connection leading to many dead ends. Here high $\sigma$ is due to the low crack spacing. Closer to $Y \sim 3$ cracks become wider and better connected, but sparse. So whether to choose $Y \sim 1$ or $Y \sim 3$ for an application has to be based on the requirement, whether dead-ends in the conducting network are useful ( e.g., for efficient charge carrier collection in solar cells) or not.

\subsection{Effect of distribution in $Y$ on conductivity}

In the simulation model, the stiffness of the Hookean springs, $Y$, characterizes the stiffness of the sample under study. A single value of $Y$ for all springs implies that the sample is made up of an ideally pure constituent. The pure constituent has particles of identical composition and size and a pure homogeneous solvent. Addition of particles of the same size, but different compositions, or vice-versa, induces an inhomogeneity manifested as a range in $Y$ values. We have introduced a deviation $\delta Y$ about the mean value of $Y$ and studied the effect 

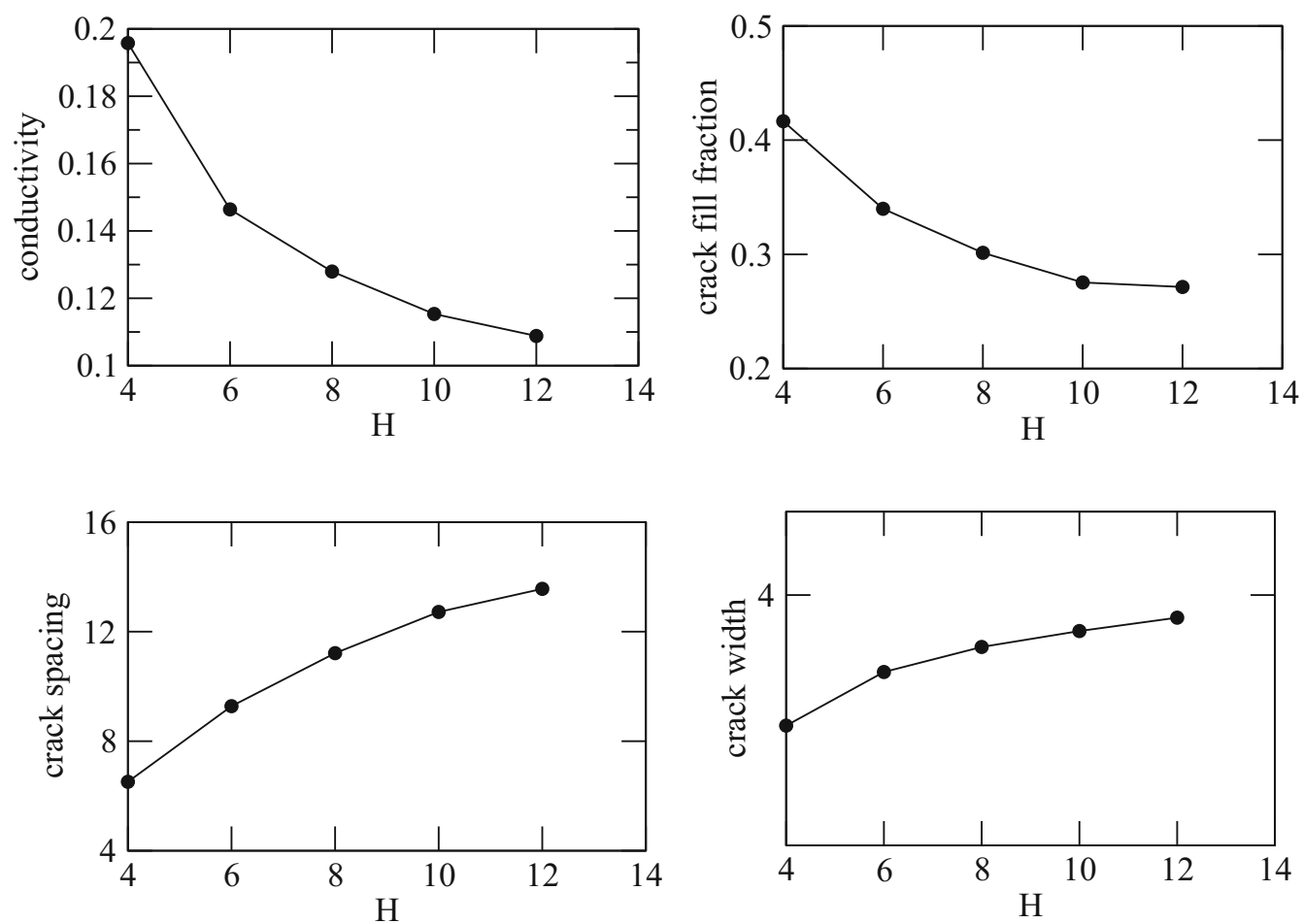

Figure 7. Different crack properties for varying thickness.

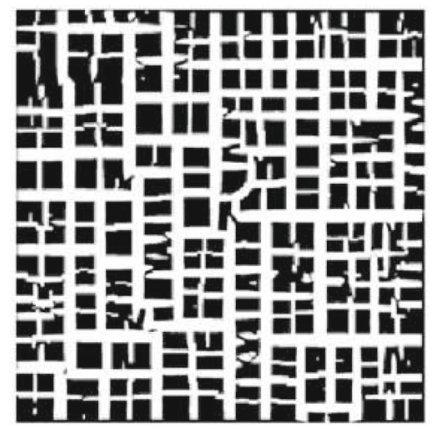

$\mathrm{Y}=0.2$

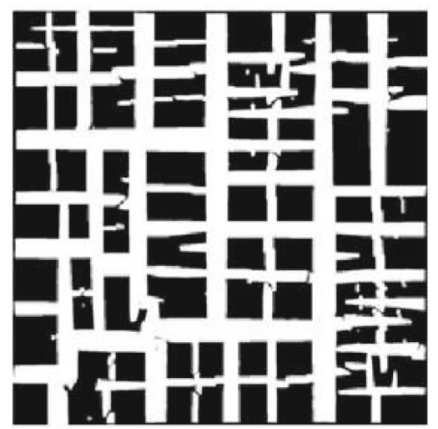

$\mathrm{Y}=1.0$

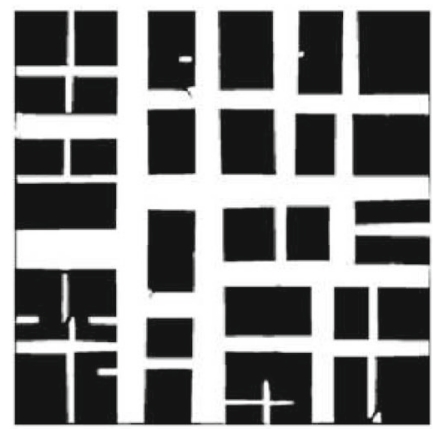

$\mathrm{Y}=\mathbf{5 . 0}$

Figure 8. Varying $Y$ shows marked differences in the crack pattern.

on the conductivity of the cracked and dried sample after desiccation. Simulation results show a very strong effect of the inhomogeneity on the crack pattern.

Figure 10a shows that with a deviation of $\Delta Y$, the nearly straight and well-defined cracks tend to become disordered and random. In addition, the number of cracks spanning the sample and hence, the conductivity decrease. In fact, for a deviation $>3 \%, \sigma$ tends to 0 , so the variation shown in the figures are for a very small range, where $\sigma>0$. A similar trend was observed on adding $\mathrm{Al}_{2} \mathrm{O}_{3}$ into $\mathrm{TiO}_{2}$. The cracks become disconnected and non-linear on the addition of $\mathrm{Al}_{2} \mathrm{O}_{3}$ as shown in figure 10b. Similar observations were stated in ref. [13], where we reported that cracks formed on pure
Laponite ${ }^{\circledR}$ clay films were straight, while film obtained from a mixture of clay and polymer showed disconnected and random cracks. The fractal dimensions of the experimentally obtained cracks have been calculated by box counting. It is found that for the sample with $\delta Y=0$, the fractal dimension is 1.66 , while that for $\delta Y= \pm 0.3 \%$ is 1.71 indicating that the fractal nature increases with the deviation, implying a rougher crack path. There are several works which corroborate this observation. Urabe and Takesue [32] observed meandering cracks in composites, which inhibit failure by crack formation. Kiruthika et al [18] reported that cracks on nanofilms made of uniform size particles were observed to be straight and connected, while that of non-uniform size showed 

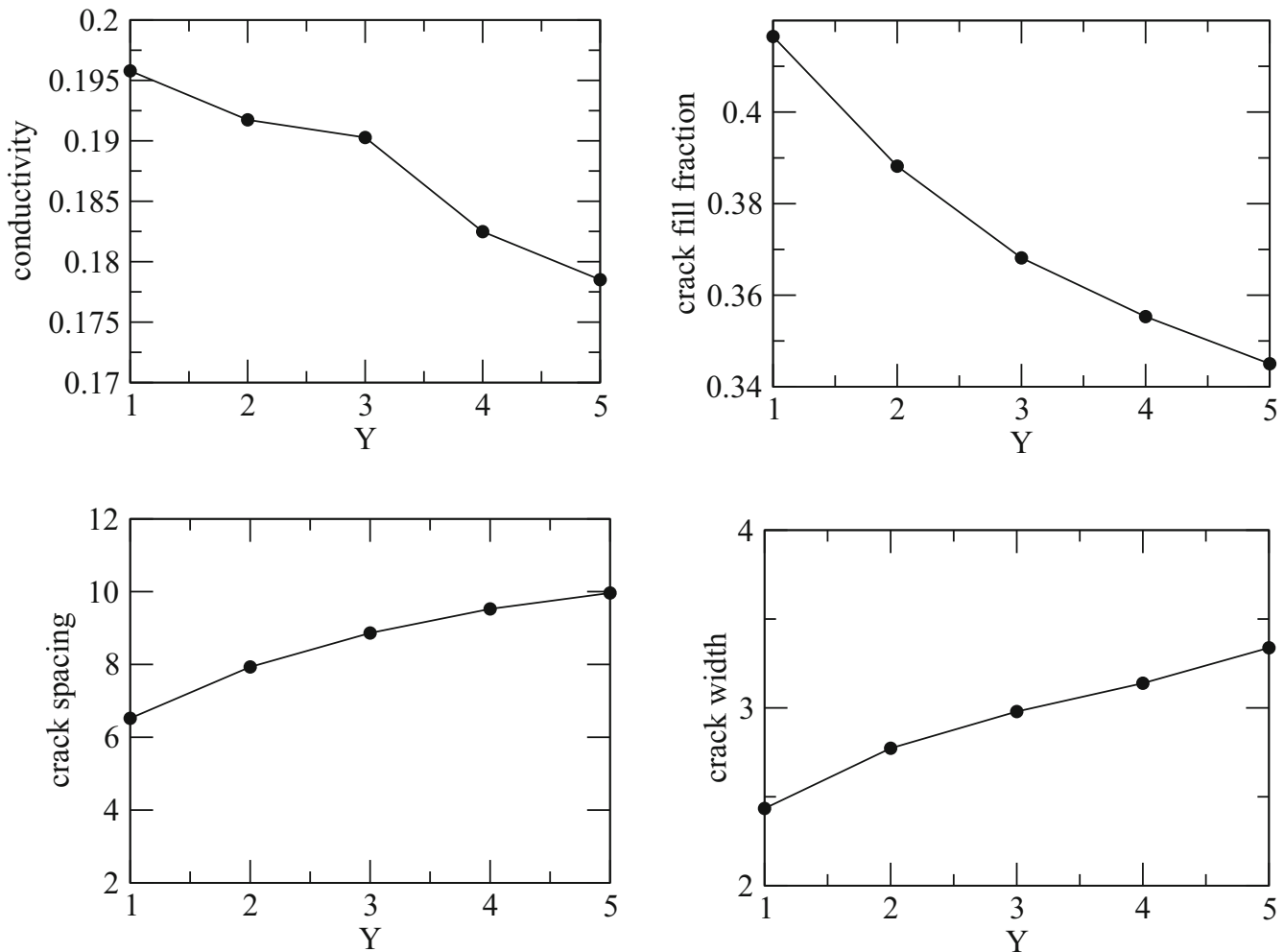

Figure 9. Variations in crack properties with $Y . Y$ values in arbitrary units.

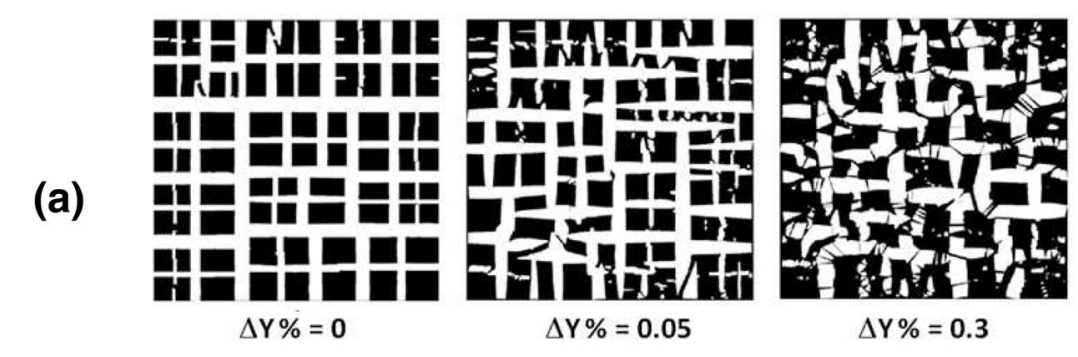

(b)
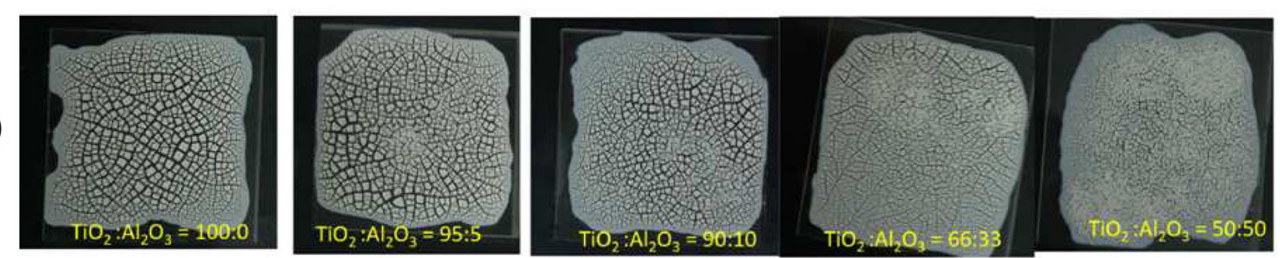

(c)
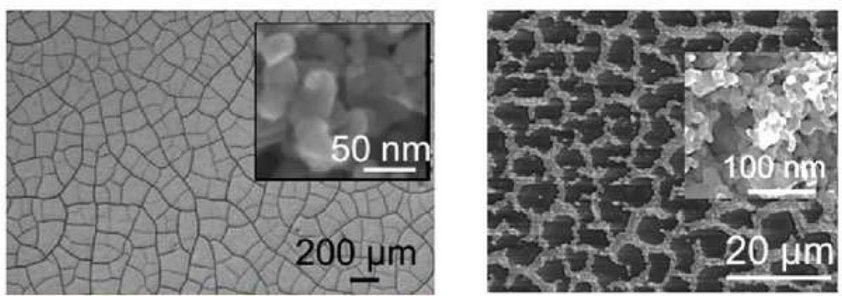

Figure 10. (a) Sample images for $\Delta Y \%$. (b) Variations in crack geometry of $\mathrm{TiO}_{2}$ colloidal film on addition of $\mathrm{Al}_{2} \mathrm{O}_{3}$ causing deviation $\Delta Y$. (c) Crack geometry on the film obtained from $\mathrm{TiO}_{2}$ nanoparticles of (i) uniform size of $25 \mathrm{~nm}$ and (ii) non-uniform size with a mean of $16 \mathrm{~nm}$. 

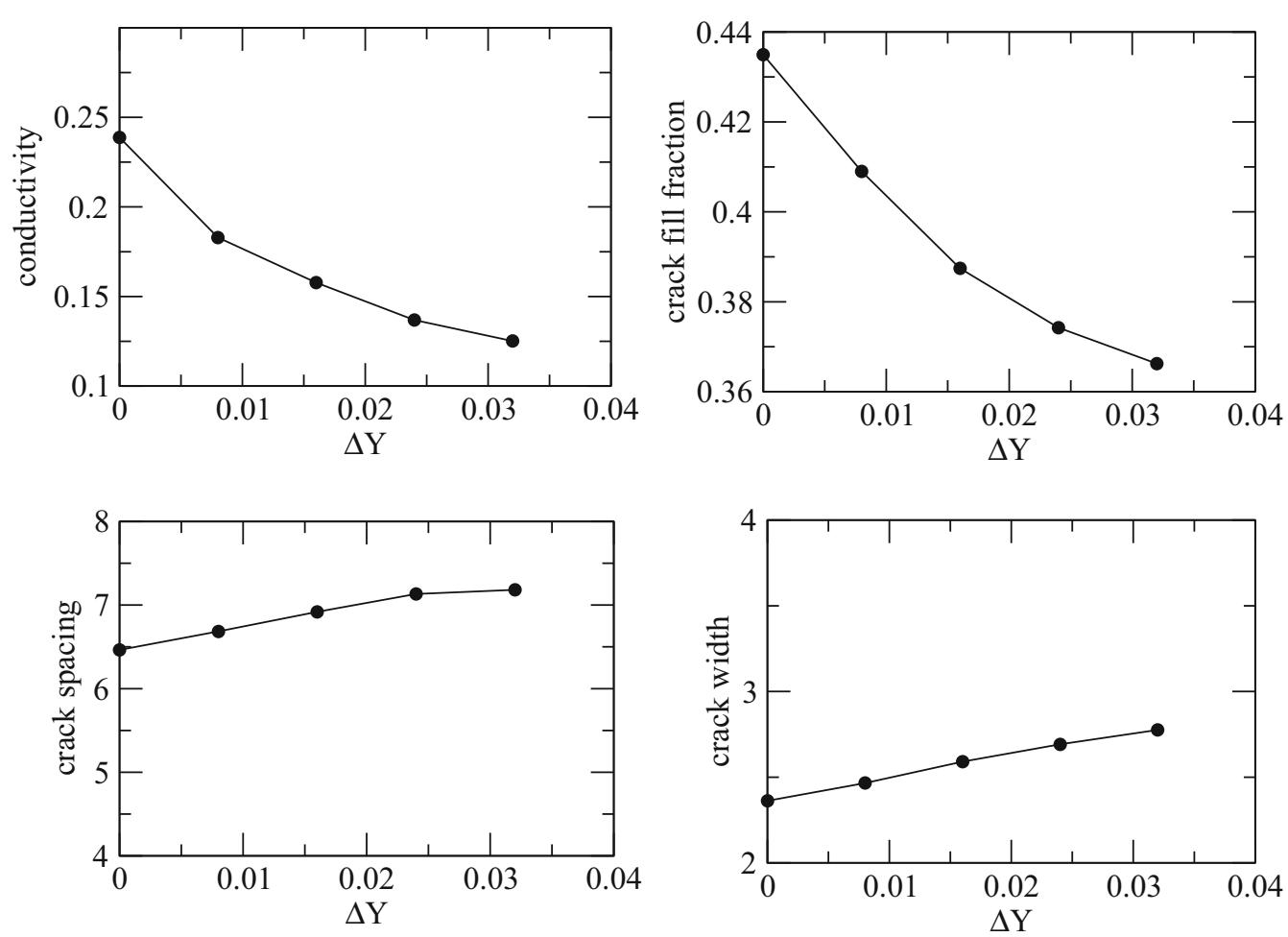

Figure 11. Variations in crack properties for $\Delta Y \% . \Delta Y \%$ in arbitrary units.

randomness, lower connectivity and increased fractal nature (figure 10c). Figure 11a shows the variation of conductivity with $\delta Y$. It is observed that there is a substantial fall in the conductivity even for a small deviation from $Y$. At $\delta Y=0$, the maximum conductivity is obtained. This value reduces to half of the maximum value at $\delta Y= \pm 3.2 \%$.

Figure 11 shows that the crack fill factor closely follows the decreasing trend in the conductivity curve, while both crack width and spacing increase with $\delta Y$. The observations can be understood by scrutinizing figure 10 , where we see that the cracks for higher $\delta Y$ though wide, are mostly disconnected and do not contribute to $\sigma$. Therefore, inhomogeneity in the sample is clearly not desirable for TCE fabrication.

\subsection{Variations of breaking threshold}

Figure 12 shows no marked differences for simulation experiments done for $S=0.025$ and $S=0.1$, here, crack spacing is measured from one crack boundary to the adjacent crack boundary. Figure 13 shows that conductivity and fill factor decrease very slightly and linearly by $<20 \%$ as $S$ increases, while crack width and spacing decrease by a negligible amount of $S$. On the whole, there is no significant variation for the crack properties with $S$. This would imply that the stiffness or Young's modulus of the sample plays a more important role in crack morphology than the fracture toughness for a pure material.

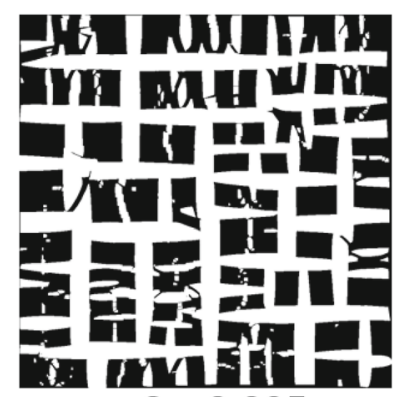

$\mathrm{S}=0.025$

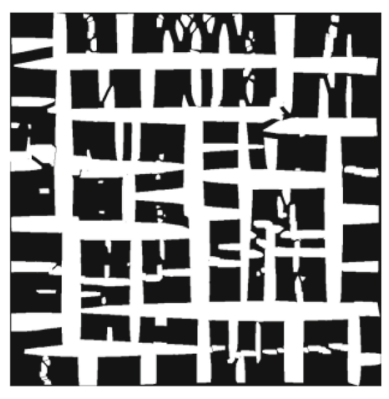

$S=0.1$
Figure 12. Variation in breaking threshold $S$ of the springs.

\subsection{Variations of drying rule through $b$ and $r$}

As discussed in section 2.3, $b$ and $r$ jointly control the drying rate. Higher $b$ and lower $r$ lead to more rapid drying, as they occur in the combination $b / r^{n}$ in equation (1). We argued that the form of the empirical rule implies that $b$ can be correlated with the ambient conditions, rather than material properties and vice versa for $r$. Figure 14 shows typical sample images that show cracks become more well-connected with an increase in $b$, i.e., with lower relative humidity and higher temperature.

Plots in figure 15 show that conductivity and fill factor increase with $b$, while the crack spacing decreases with $b$. The crack width, however, increases slightly with $b$, which is not desirable, if it goes beyond the invisibility limit for TCS. 

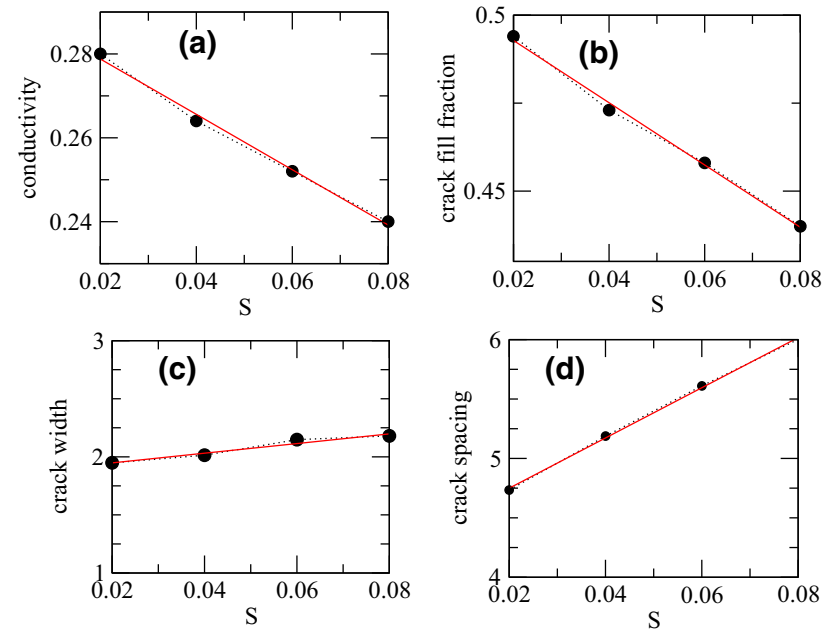

Figure 13. Variation of crack template properties with breaking threshold $\mathrm{S}$. Breaking threshold $\mathrm{S}$ is in arbitrary units. The template parameters studied are (a) conductivity, (b) crack fill fraction, (c) crack width and (d) crack spacing.

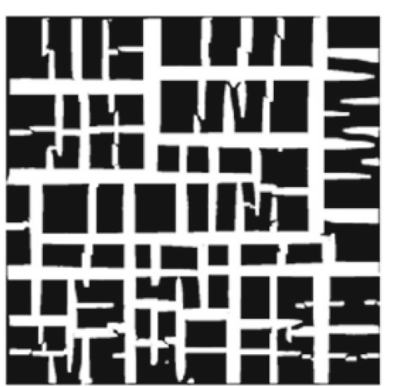

$b=0.04$

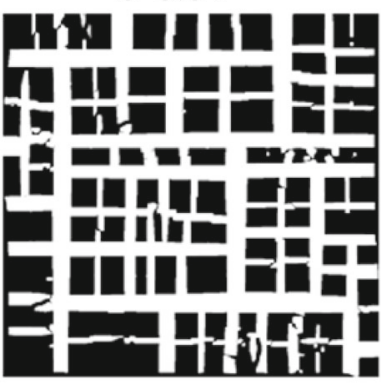

$r=1.07$

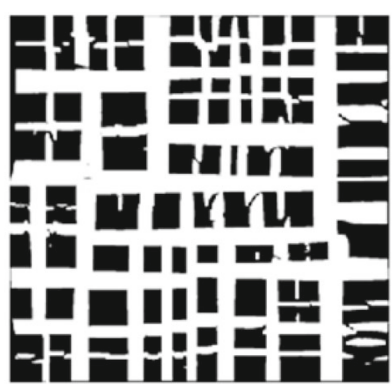

b $=0.06$

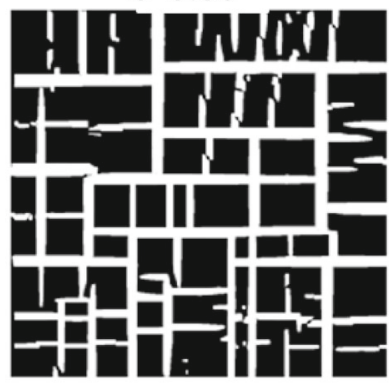

$r=1.1$
Figure 14. Images for variation in $b$ and $r$ used in the drying rule.

The increase in $r$ naturally has a reverse effect to that caused by $b$ since it is in the denominator of equation (1). According to our analysis, rapid drying due to smaller $r$ implies using a more volatile solvent. Clearly, the influence of drying rate is very strong on the crack pattern, so $b$ and $r$ have to be tuned simultaneously and judiciously to optimize conductivity and transparency for application in devices.

\section{Discussion}

We have modelled a desiccating colloidal suspension through a spring network model in $3 \mathrm{D}$. We investigated the effect of various parameters that control crack geometry. When used as a template for fabricating TCS, the parameters should be such that good conductivity and high transparency are obtained. However, this is not enough. For certain device applications, a dense network where there are fewer areas with no cracks is preferred compared to a pattern with equal conductivity and transparency, but large areas with no crack. Depending on the particular device, where the TCS is to be used, dead end or 'hanging bond' cracks in the network may or may not be desirable. For solar cell applications, a dense network with some hanging bonds may be useful, but if the conductivity is the prime concern, hanging bonds will unnecessarily reduce transparency, without contributing to conduction.

Since the model parameters can be related to measurable physical properties of the materials and ambient conditions during drying, the simulation gives us an idea about the types of materials and conditions to be implemented for the best results for a certain device application. The most striking result is the necessity for homogeneity in the material. Addition of even a small amount of impurity to the uniform composition of a desiccating material causes a marked change in crack geometry. Hence, for any non-zero deviation $\Delta Y$, randomness in the crack pattern comes into play and conductivity falls substantially. Figures 10 and 11 show a fall in conductivity on introducing slightest polydispersity. Also, a lower $\Delta Y$ yields lower values for crack width and spacing, which is the required condition for high transparency and high connectivity [20].

The choice of the value of $Y$, i.e., the stiffness of the colloidal paste is also very important for determining the optimum condition of large conductivity and transparency. At a low value of $Y=1$ in our simulation, the conductivity is maximum with a fill fraction quite high (figure 9). The crack width and spacing are also low. However, as discussed in section 4, it is possible to have a relatively large conductivity with a low fill factor, i.e., more transparency for $Y=3$.

We show that crack width and spacing increase with the thickness of a film, as previously found in simulation and experiments $[9,10]$. The conductivity increases with decreasing thickness $H$ of the suspension as long as $H$ is above the critical value for the formation of a connected crack network.

Variation in breaking threshold (figure 13) shows that all the properties considered scale weakly and linearly with $S$. Crack width and crack fill factor change only by a negligible amount as $S$ varies. We may infer that if the limit is not infinitely large as in the case of polymers, which are very difficult to break, there will be no significant change in crack morphology with $S$.

Besides materials, the drying conditions are to be chosen carefully as well. In the same time interval, a faster desiccation rate leads to a greater stress builds up within the system. As a result, a spring is stretched faster before it reaches its yielding point. Two materials having approximately the same $Y$ can 

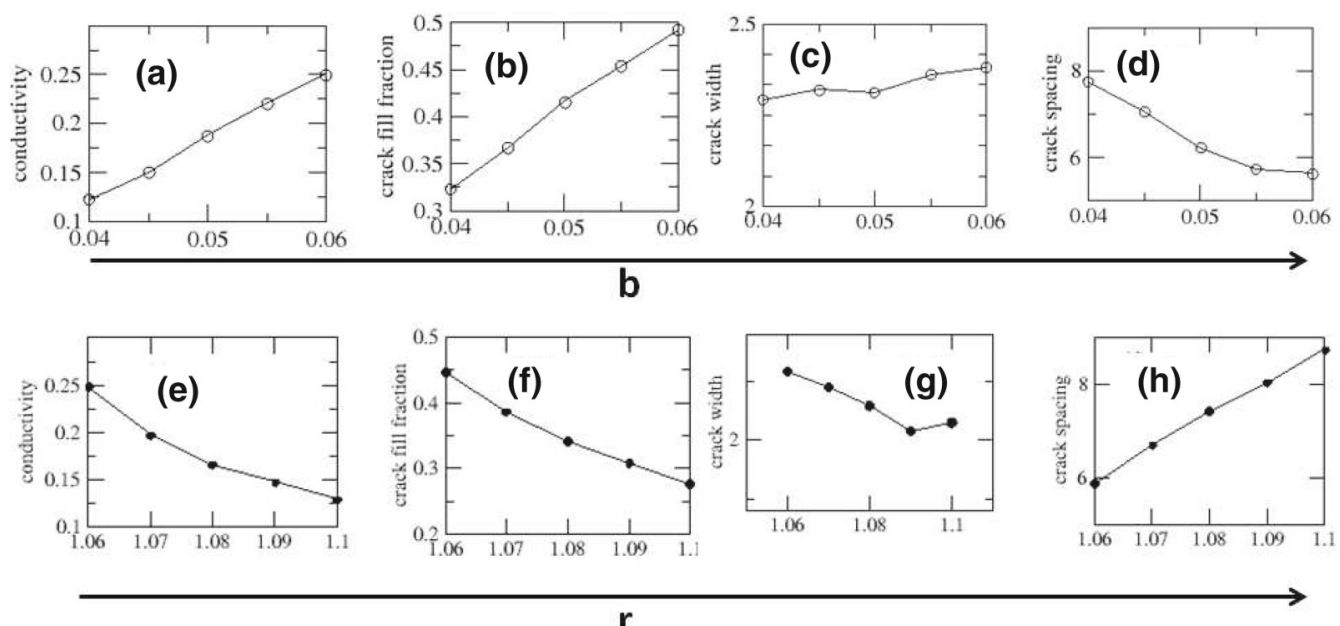

Figure 15. (a-d) Variations of crack parameters with variable $b$ used in the drying rule. (e-h) Variations of crack parameters with variable $r$ used in the drying rule. $b$ and $r$ are in arbitrary units.

Table 1. Simulation parameters: sample height $(H)$, stiffness $Y$ and its range $\Delta Y$ for an inhomogeneous sample, strain threshold $S$, drying parameters $b$ and $r$ and their limits of variation. All entries are in arbitrary units.

\begin{tabular}{lcccccc}
\hline Parameters & Height, $H$ & Stiffness, $Y$ & $Y$ Range, $\Delta Y$ & Strain threshold, $S$ & RH/temp, $b$ & Volatility, $r$ \\
\hline Fixed value & 4 & 1 & 0 & 0.075 & 0.05 & 1.06 \\
Variation limits & $4-12$ & $0.2-5.0$ & $0-0.3 \%$ & $0.025-0.100$ & $0.04-0.06$ & $1.06-1.10$ \\
\hline
\end{tabular}

behave very differently, if one desiccates faster than the other. We observe that with higher drying rate (controlled by $b$ and $r$ ), well-connected cracks with less crack spacing form yield high conductivity. However, on the other hand, the increase in crack width beyond a certain limit is not favourable as it tends to decrease transparency.

\section{Conclusions}

We have developed a spring-network model in 3D and simulated the formation of a conducting network on it, reproducing the technique of vapour-deposition of metal along the cracks created by drying a colloidal film on a transparent surface [18]. The objective is to create a conducting network, which optimizes conductivity and transparency and thus, is most suitable for application as a TCE.

We have tried to correlate the model parameters to measurable physical properties of the real materials used, namely, the elasticity and composition of the colloid, the thickness of the drying layer and the rate of drying. The analysis is complicated by inter-relations between these properties and the ambient conditions, but we have identified some broad features as follows, which we expect may be used in practice to fabricate effective TCE.
1. The colloid should be homogeneous: if materials with elastic properties varying over a range are mixed, the network formed is not suitable for the application.

2. The thickness of the colloidal suspension should be as small as possible, as long as it is above the critical cracking thickness $[1,10]$.

3. For high conductivity, the stiffness of the suspension (corresponding to Young's modulus if measurable or Hamaker constant of the fluid-solid combination) should, in general, be low, but this compromises the transparency. However, since several factors in combination produce the final conduction properties of the network, it is also possible that a higher stiffness material will optimize acceptably high conductivity together with high transparency.

4. The drying rate should be chosen based on the requirement of transmittance and conductivity. Higher drying rate can be implemented through the ambient conditions, by keeping the low relative humidity and high temperature, and also by choosing a more volatile solvent. A higher drying rate leads to more conductivity, but compromises transmittance.

In this preliminary study, we state the physical properties of the material under study and ambient conditions in arbitrary 
units. This gives an idea of the effect of relative variation of these quantities on the crack pattern and hence, the features and efficacy of the TCE to be fabricated. Our future goal is to translate these results in terms of the physical properties of materials in real physical units, which may actually be used for TCE template fabrication.

\section{Acknowledgements}

Sujata Tarafdar is grateful to DST, Government of India, for financial support.

\section{References}

[1] Goehring L, Nakahara A, Dutta T, Kitsunezaki S and Tarafdar S 2015 Desiccation cracks and their patterns (New Jersey: Wiley)

[2] Bacchin P, Brutin D, Davaille A, Di Giuseppe E, Chen X D et al 2018 Eur. Phys. J. E 4194

[3] Skjeltorp A T and Paul M 1988 Nature 335424

[4] Andrade P N and Fortes M A 1988 Philos. Mag. Part B 58 1988

[5] Sircar S, Khatun T, Dutta T and Tarafdar S 2016 Indian J. Phys. 901355

[6] Kitsunezaki S 1999 Phys. Rev. E 606449

[7] Colina H and Roux S 2000 Eur. Phys. J. E 1189

[8] Somasri H, Sudeshna S, Tajkera K, Moutushi D C, Giri A, Sanat K et al 2016 RSC Adv. 664297

[9] Sadhukhan S, Prehl J, Blaudeck P, Hoffmann K H, Dutta T and Tarafdar S 2008 Eur. Phys. J. E 27391

[10] Khatun T, Dutta T and Tarafdar S 2015 Eur. Phys. J. E 3883

[11] Hirota K, Tanoue Y and Kaneko T 2000 Vis. Comput. 16 371
[12] Halász Z, Nakahara A, Kitsunezaki S and Kun F 2017 Phys. Rev. E 96033006

[13] Nag S, Sinha S, Sadhukhan S, Dutta T and Tarafdar S $2010 \mathrm{~J}$. Phys.: Condens. Matter 22015402

[14] Sadhukhan S, Dutta T and Tarafdar S 2011 Physica A: Stat. Mech. Appl. 390731

[15] Dutta T and Tarafdar S 2014 J. Mater. Sci. 497507

[16] Vogel H J, Hoffmann H and Roth K 2005 Geoderma 125203

[17] Amarasiri Aruna L, Kodikara Jayantha K and Susanga C 2011 Int. J. Numer. Anal. Methods Geomech. 3582

[18] Kiruthika S, Rao K D M, Kumar A, Gupta R and Kulkarni G U 2014 Mater. Res. Express 1026301

[19] Rao K D M, Gupta R and Kulkarni G U 2014 Adv. Mater. Interfaces 1140009

[20] Kumar A and Kulkarni G U 2016 J. Appl. Phys. 119015102

[21] Kumar A 2017 J. Appl. Phys. 121014502

[22] Kumar A, Pujar R, Gupta N, Tarafdar S and Kulkarni G U 2017 Phys. Lett. 111013502

[23] Sadhukhan S, Majumdar S R, Mal D, Dutta T and Tarafdar S 2007 J. Phys.: Condens. Matter 19356206

[24] Sadhukhan S, Dutta T and Tarafdar S 2007 J. Stat. Mech. Theory Exp. 06 P06006

[25] Sadhukhan S, Dutta T and Tarafdar S 2007 Modelling Simul. Mater. Sci. Eng. 15773

[26] Roth M, Schilde C, Lellig P, Kwade A and Auernhammer G K 2012 Eur. Phys. J. E 35124

[27] Hay J 2009 Exp. Tech. 3366

[28] Hull D 1999 Fractography: observing, measuring and interpreting fracture surface topography (Cambridge: Cambridge University Press) ISBN 0521646847, 9780521646840

[29] Sadd M H 2005 Elasticity: theory, applications and numerics (Amsterdam: Elsevier) ISBN 0-12-605811-3

[30] Bohn S, Pauchard L and Couder Y 2005 Phys. Rev. E 71046214

[31] Tirumkudulu Mahesh S and Russel William B 2005 Langmuir 214938

[32] Urabe C and Takesue S 2010 Phys. Rev. E 82016106 